\title{
Dentatorubro-Pallidoluysian Atrophy (DRPLA) among 700 Families with Ataxia in Brazil
}

\author{
Pedro Braga-Neto ${ }^{1,2}$ • José Luiz Pedroso ${ }^{3}$ - Gabriel Vasata Furtado ${ }^{4,5}$. \\ Tailise Conte Gheno $^{4,5}$ • Maria Luiza Saraiva-Pereira ${ }^{4,5,6}$ • Laura Bannach Jardim ${ }^{5,6,7}$. \\ Orlando G. P. Barsottini ${ }^{3}$ • on Behalf of Rede Neurogenetica
}

Published online: 21 April 2017

(C) Springer Science+Business Media New York 2017

\begin{abstract}
Dentatorubro-pallidoluysian atrophy (DRPLA) is a spinocerebellar ataxia (SCA) very rare in non-Asian populations. To date, DRPLA was undetected in the general Brazilian population. Adult-onset ataxic patients have been recruited from several Brazilian neurology and neurogenetics centers. CAG lengths at SCA1, SCA2, SCA3/MJD, SCA6, SCA7, SCA12, SCA17 and DRPLA associated genes, and ATTCT expansions at SCA10 gene were studied. A single DRPLA case detected is reported. Proband was a 69-year-old Brazilian woman of mixed ancestry, with a late-onset pure ataxia: her alleles at the associated gene, ATN1, presented 14/52 CAG repeats. History of gait ataxia and dementia was observed in two out of six siblings but was absent in her parents. This was the single DRPLA diagnosis obtained from 700 Brazilian unrelated cases with adult-onset ataxia, 487 of them with clear autosomal dominant inheritance. DRPLA accounted for $0.14 \%$ of all adult-onset ataxia cases and for $0.2 \%$ of families with autosomal dominant inheritance.
\end{abstract}

Pedro Braga-Neto and José Luiz Pedroso contributed equally to this work.

Electronic supplementary material The online version of this article (doi:10.1007/s12311-017-0862-9) contains supplementary material, which is available to authorized users.

Laura Bannach Jardim

ljardim@hcpa.edu.br

1 Centro de Ciências da Saúde, Universidade Estadual do Ceará, Fortaleza, Brazil

2 Departamento de Medicina Clinica, Faculdade de Medicina, Universidade Federal do Ceará, Fortaleza, CE, Brazil

3 Departamento de Neurologia, Unidade de Ataxia, Universidade Federal de São Paulo, São Paulo, SP, Brazil
Normal CAG repeats at ATN1 had a median (range) of 14 (5-20) repeats in other 410 Brazilian chromosomes. DRPLA is quite rare in Brazilian SCA families, which is consistent with the lack of large normal alleles in our population.

Keywords Dentatorubro-pallidoluysian atrophy · Epidemiology $\cdot$ Polyglutamine disorders $\cdot$ Spinocerebellar ataxias

\section{Introduction}

Dentatorubro-pallidoluysian atrophy (DRPLA) is an autosomal dominant spinocerebellar ataxia (SCA) caused by an abnormal expansion of a CAG repeat at ATN1 gene that is located on chromosome 12p13 [1]. Expanded CAG tracts (CAGexp) of this gene show 48 or more repeats, and the age of onset and clinical severity is inversely correlated with
4 Programa de Pós-Graduação em Genética e Biologia Molecular, Universidade Federal do Rio Grande do Sul, Porto Alegre, Brazil

5 Serviço de Genética Médica e Laboratório de Identificação Genética, Hospital de Clínicas de Porto Alegre, Rua Ramiro Barcelos 2350, Porto Alegre 90035-003, Brazil

6 Departamento de Bioquímica, Universidade Federal do Rio Grande do Sul, Porto Alegre, Brazil

7 Departamento de Medicina Interna, Universidade Federal do Rio Grande do Sul, Porto Alegre, Brazil 
length of expansion [2]. DRPLA is very rare in Latin America. For instance, frequency of DRPLA was estimated to be $3.1 \%$ among SCAs in Venezuela [3], while only one single case has been described so far in Brazil in an individual of Japanese ancestry [4].

Different phenotypes including chorea, cerebellar ataxia, and progressive myoclonic epilepsy have been described in DRPLA, and there is a close relationship with age at onset and CAGexp length. The cardinal feature of juvenile presentation of the disease ( $<25$ years) is a progressive myoclonic epilepsy, whereas in the late-onset DRPLA ( $>40$ years), progressive ataxia, dementia, and chorea are the core findings. DRPLA is one of the several SCAs due to intraneuronal polyglutamine aggregation. As observed in SCA2, SCA3/Machado Joseph disease, and SCA17, phenotype might change substantially according to age at onset [5].

This work aimed to describe the first case of DRPLA among a large cohort of ataxic individuals from Brazil, to estimate its frequency among other SCAs diagnosed by the same laboratory, and to describe ATN1 CAG repeats distribution in the Brazilian population.

\section{Methods}

Adult-onset ataxic patients have been recruited from several neurology and neurogenetics centers from Brazil as previously described. They were included in a SCA screening panel when appropriated and following obtaining written informed consent [6]. Relative frequency of DRPLA among SCAs previously investigated by the same laboratory (Rede Neurogenetica, Laboratorio de Identificacao Genetica, Hospital de Clinicas de Porto Alegre) was obtained. Finally, distribution of the CAG repeats at 411 normal ATN1 alleles from other Brazilian individuals was depicted. The present work has been approved by the Ethics Committee from the institution at which the work was performed-Comissão de Ética em Pesquisa do Hospital de Clínicas de Porto Alegrewhich follows the Code of ethics of the World Medical Association (Declaration of Helsinki) and the standards established by the author's Institutional Review Board and granting agency.

\section{Results and Case Report}

The proband was a 69-year-old Brazilian woman of mixed (Iberic, Amerindian, and African) ancestry with a 9-year history of progressive gait ataxia. Family history was remarkable for two affected siblings (Fig. 1a). Both had motor and cognitive impairment, but were not evaluated. Neurological examination disclosed a pure cerebellar syndrome, with predominant axial involvement (Video). Scale for the assessment and rating of ataxia (SARA) was 10 and international cooperative ataxia rating scale (ICARS) was 18 . Cognition and mental status were normal, without any evidence of psychiatric disturbance. Mini mental state examination was 27/30 and considered normal according to studies of Brazilian population [7]. There were no abnormal involuntary movements. Deep tendon reflexes, cranial nerve, and sensorial examination were normal. Brain magnetic resonance imaging showed mild cerebellar atrophy (Fig. 1b). Genetic investigation was performed for other genetic ataxias, such as Friedreich ataxia, SCA1, SCA2, SCA3, SCA6, SCA7, SCA10, SCA12, SCA17, and Huntington disease, according to methods described elsewhere $[6,8]$ and results for all this evaluations were negative. Molecular analysis showed that ATN1 alleles presented 14/52 CAG repeats, which confirms DRPLA diagnosis.

By June 2016, 700 index cases presenting adult-onset ataxia were investigated in the same laboratory where the diagnosis was accomplish, by studying nucleotide repeats associated to SCA1, SCA2, SCA3/MJD, SCA6, SCA7, SCA10, SCA12,
Fig. 1 Pedigree of the present DRPLA case (a). Brain MRI shows mild cerebellar and global cerebral cortex atrophy (b)

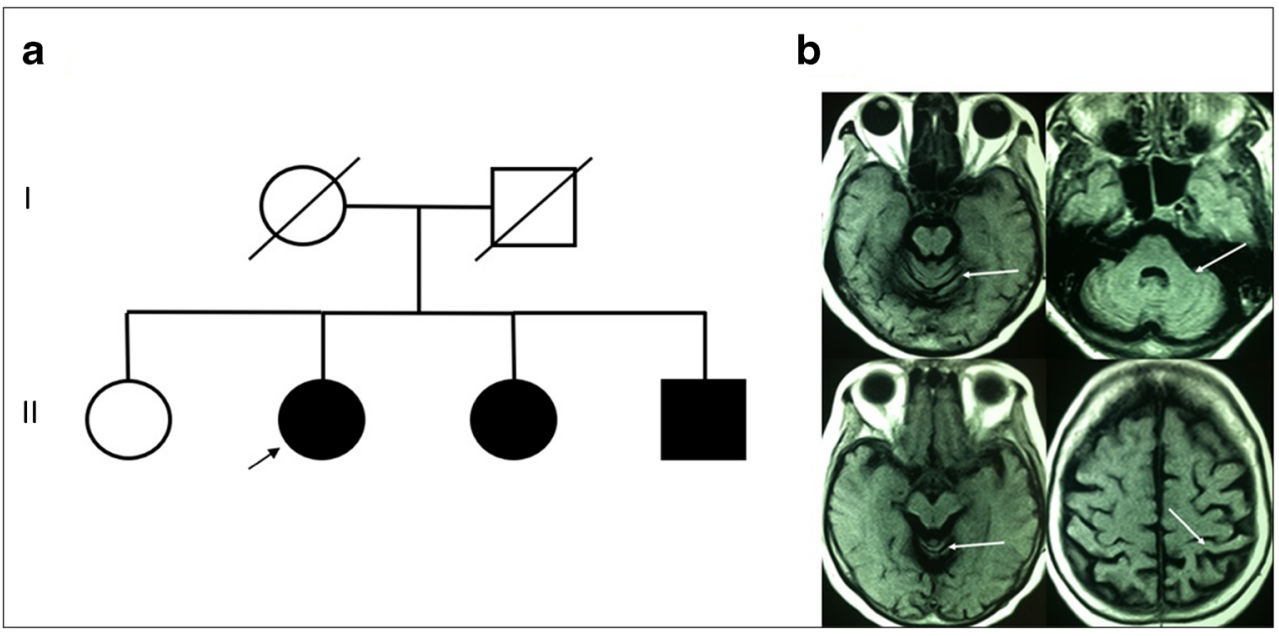


Table 1 Relative frequency of spinocerebellar ataxias due to expanded repeats, among the present case series of ataxic Brazilian individuals studied by Rede Neurogenetica

\begin{tabular}{lcc}
\hline Molecular diagnosis & $\begin{array}{c}\text { Index cases with autosomal } \\
\text { dominant inheritance (SCAs) }\end{array}$ & All index cases \\
\hline Total $N$ of SCAs & $487(100 \%)$ & $700(100 \%)$ \\
SCA3/MJD & $261(53.6 \%)$ & $265(37.8 \%)$ \\
SCA2 & $56(11.5 \%)$ & $59(8.4 \%)$ \\
SCA7 & $22(4.5 \%)$ & $22(3 \%)$ \\
SCA1 & $21(4.3 \%)$ & $21(3 \%)$ \\
SCA10 & $11(2.2 \%)$ & $13(2 \%)$ \\
SCA6 & $6(1.2 \%)$ & $6(0.8 \%)$ \\
DRPLA & $1(0.2 \%)$ & $1(0.14 \%)$ \\
SCA12 & $1(0.2 \%)$ & $1(0.14 \%)$ \\
SCA17 & 0 & 0 \\
Without diagnosis & $109(22.3 \%)$ & $313(44.7 \%)$ \\
\hline
\end{tabular}

SCA spinocerebellar ataxias, MJD Machado-Joseph disease, DRPLA dentatorubral-pallidoluysian atrophy

SCA17, and DRPLA. Some index cases presented a clear autosomal dominant inheritance (487/700), while others were isolate, sporadic individuals without a positive family history (213/700). Table 1 depicts the overall results. Geographical origins varied between different parts of the country, with majority coming from South Brazil [6]. Allele distribution of 176 normal CAG repeats at ATN1 in Brazilian individuals has been formerly documented [6]. Following that, additional subjects were added and 410 alleles were measured, with a median (range) of 14 (5-20) repeats (data until June 2016). Only $4.3 \%$ of these CAG alleles show more than 17 repeats (Fig. 2).

\section{Discussion}

DRPLA is an extremely rare form of SCA in Brazil. This was our first case diagnosed and, as far as we are aware, the first case from the general Brazilian population. Patient presented a late-onset form of ataxia, and her diagnosis was achieved after excluding all other forms of SCAs due to expanded nucleotide repeats. The only single Brazilian case previously diagnosed was a man of Japanese ancestry that presented progressive myoclonic epilepsy starting at 17 years of age [4]. Other large case series of SCAs from Brazil did not detect DRPLA [9-11]. Curiously, the two DRPLA individuals diagnosed in Brazil showed the two phenotypic extremes, the juvenile- and the late-onset forms, illustrating that clinical manifestations are independent from geographical origins.

A significant proportion of cases of DRPLA among SCAs in general were found in Japan (7-20\%), Singapore (6\%), and Korea (3\%) [12]. Despite being considered a rare disease in non-Asian populations, DRPLA patients have been eventually reported in several countries, particularly in Europe and America. DRPLA was estimated to affect 0.25 to $1 \%$ of European patients with sporadic or autosomal dominant
Fig. 2 Distribution of CAG repeats at normal ATN1 alleles obtained from 410 Brazilian chromosomes

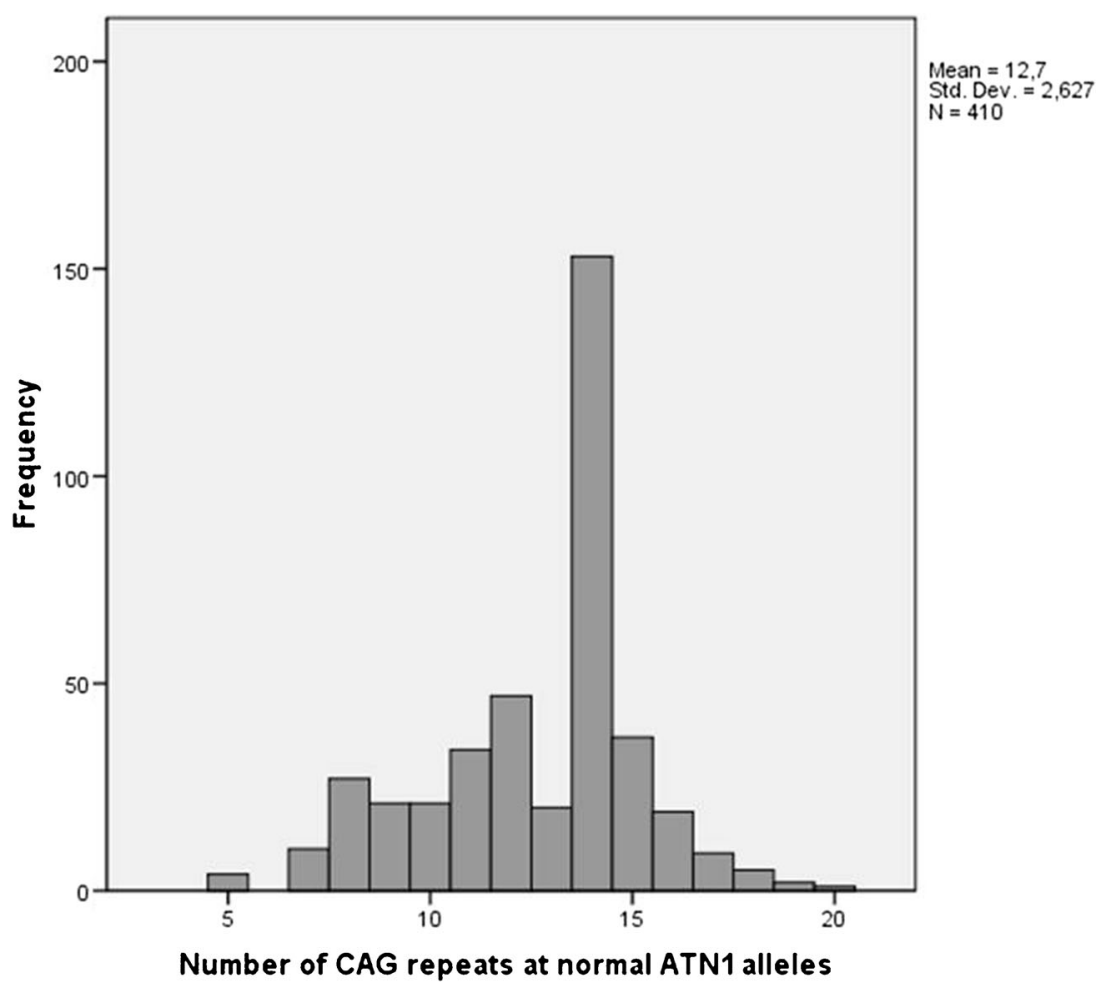


cerebellar ataxia from series with 116 to 809 index cases [1,2, 13]. A large nationwide, population-based survey improved these figures, identifying DRPLA in $4 \%$ of Portuguese SCA families [14]. Brazilian population is greatly mixed and it is hard to state that the present DRPLA expansion would have a Portuguese, Amerindian, or African origin. Regarding the prevalence of DRPLA in other countries of South America, we only found an epidemiologic study of SCA in Venezuela describing an estimated prevalence of $3.1 \%$ [3].

Isolated, sporadic forms of DRPLA have been described in both Asian and non-Asian families [1, 15]. There are a number of reasons for this, including unknown family history and early death of affected ancestors. The relative contribution of de novo CAG expansions is uncertain [16]. Normal alleles at ATN1 range from 6 to 35 CAG repeats; those with 17 to 35 repeats are uncommon and considered mutable normal alleles (mNA) $[17,18]$. Higher frequencies of mNA in a given population have been related to relatively higher rates of DRPLA. For instance, mNA occurred in $24 \%$ of Japanese, in $10 \%$ of French, and in $4.3 \%$ of the present Brazilian chromosomes. Conversely, DRPLA corresponds to $7-2,0.25$, and $0.20 \%$ of SCA families in Japan, France, and Brazil, respectively $[1,2,14]$.

Clinical presentation is strongly correlated to the length of expanded CAG repeats [18]. Because this relationship was questioned by small non-Asian series, a systematic review evaluated the clinical and genetic profile of 183 non-Asian DRPLA patients. Clinical phenotype and mode of presentation in non-Asian patients were also highly dependent on CAGexp [15]. Asian and non-Asian affected individuals with more than 65 CAGexp present a juvenile onset and epilepsy as the main finding, whereas those with fewer than 65 CAGexp repeats are non-juvenile cases presenting heterogeneous features that include ataxia, dementia, chorea, and psychiatric disease $[15,18]$. The CAGexp of our present case -52 repeats - was within the range of late-onset forms. After 9 years from the beginning of symptoms, she presented a pure form of ataxia. Unfortunately, we were unable to examine her ataxic and demented sibs in order to compare their CAGexp lengths and to correlate them with their more severe manifestations.

This is the first report to date on the occurrence of DRPLA in a Brazilian individual without Japanese ancestry, and also the first report on frequency of DRPLA among a large Brazilian series of SCA families and adult-onset isolated cases of ataxia. The phenotype of a late-onset pure ataxia should be considered in non-Asian DRPLA patients. Taken into account this preliminary observation, we recommend that patients with undetermined SCA should be tested for DRPLA.

Acknowledgement The authors would like to thank the families who agreed to participate in this study.
Authors Contribution Conception or design of the work: PBN, JLP, MLSP, LBJ, OB. Acquisition, analysis, or interpretation of data for the work: PBN, JLP, GVF, TCG, MLSP, LBJ, OB.

Drafting the work or revising it critically for important intellectual content: PBN, JLP, GVF, TCG, MLSP, LBJ, OB.

Final approval of the version to be published: PBN, JLP, GVF, TCG, MLSP, LBJ, OB.

Agreement to be accountable for all aspects of the work in ensuring that questions related to the accuracy or integrity of any part of the work are appropriately investigated and resolved: PBN, JLP, GVF, TCG, MLSP, LBJ, OB.

\section{Compliance with Ethical Standards}

Conflict of Interests The authors declare that they have no conflict of interest.

Funding This work was supported by the following Brazilian agencies: FAPERGS — Fundação do Amparo à Pesquisa do Rio Grande do Sul—by the project 0700832 EDITAL PPSUS FAPERGS 006/2006; FIPEHCPA-Fundo de Incentivo à Pesquisa do Hospital de Clínicas de Porto Alegre-by the Project GPPG HCPA 06-384; and by INAGEMP - Instituto Nacional de Genética Médica Populacional. PBN, MLSP, and LBJ were supported by CNPq - Conselho Nacional de Desenvolvimento Científico e Tecnológico.

\section{References}

1. Filla A, Mariotti C, Caruso G, Coppola G, Cocozza S, Castaldo I, et al. Relative frequencies of CAG expansions in spinocerebellar ataxia and dentatorubropallidoluysian atrophy in 116 Italian families. Eur Neurol. 2000;44(1):31-6.

2. Le Ber I, Camuzat A, Castelnovo G, Azulay JP, Genton P, Gastaut $\mathrm{JL}$, et al. Prevalence of dentatorubral-pallidoluysian atrophy in a large series of white patients with cerebellar ataxia. Arch Neurol. 2003;60(8):1097-9.

3. Paradisi I, Ikonomu V, Arias S. Spinocerebellar ataxias in Venezuela: genetic epidemiology and their most likely ethnic descent. J Hum Genet. 2016;61(3):215-22.

4. de Souza PV, Batistella GN, Pinto WB, Oliveira AS. Teaching NeuroImages: leukodystrophy and progressive myoclonic epilepsy disclosing DRPLA. Neurology. 2016;86(6):e58-9.

5. Bird TD. Hereditary Ataxia Overview. 1998 Oct 28 [Updated 2016 Mar 3]. In: Pagon RA, Adam MP, Ardinger HH, et al., editors. GeneReviews ${ }^{\circledR}$ [Internet]. Seattle (WA): University of Washington, Seattle; 1993-2016. Available from: https://www. ncbi.nlm.nih.gov/books/NBK1138/

6. de Castilhos RM, Furtado GV, Gheno TC, Schaeffer P, Russo A, Barsottini O, et al. Spinocerebellar ataxias in Brazil-frequencies and modulating effects of related genes. Cerebellum. 2014 Feb;13(1):17-28. doi:10.1007/s12311-013-0510-y.

7. Bertolucci PH, Brucki SM, Campacci SR, Juliano Y. The minimental state examination in a general population: impact of educational status. Arq Neuropsiquiatr. 1994;52(1):1-7.

8. Castilhos RM, Souza AF, Furtado GV, Gheno TC, Silva AL, Vargas FR, et al. Huntington disease and Huntington disease-like in a case series from Brazil. Clin Genet. 2014;86(4):373-7. doi:10.1111/cge. 12283.

9. Lopes-Cendes I, Teive HG, Calcagnotto ME, Da Costa JC, Cardoso F, Viana E, et al. Frequency of the different mutations causing spinocerebellar ataxia (SCA1, SCA2, MJD/SCA3 and DRPLA) in a large group of Brazilian patients. Arq Neuropsiquiatr. 1997;55(3B):519-29. 
10. Teive HA, Munhoz RP, Arruda WO, Lopes-Cendes I, Raskin S, Werneck LC, Ashizawa T. Spinocerebellar ataxias: genotypephenotype correlations in 104. Brazilian families Clinics (Sao Paulo). 2012;67(5):443-9.

11. Cintra VP, Lourenço CM, Marques SE, de Oliveira LM, Tumas V, Marques Jr W. Mutational screening of 320 Brazilian patients with autosomal dominant spinocerebellar ataxia. J Neurol Sci. 2014 Dec 15;347(1-2):375-9. doi:10.1016/j.jns.2014.10.036.

12. Serqueiros J, Martins S, Silveira I. Epidemiology and population genetics of degenerative ataxias. Handb Clin Neurol. 2012;103: 227-51. doi:10.1016/B978-0-444-51892-7.00014-0.

13. Pujana MA, Corral J, Gratacòs M, Combarros O, Berciano J, Genís $\mathrm{D}$, et al. Spinocerebellar ataxias in Spanish patients: genetic analysis of familial and sporadic cases. The Ataxia Study Group Hum Genet. 1999 Jun;104(6):516-22.

14. Coutinho P, Ruano L, Loureiro JL, Cruz VT, Barros J, Tuna A, et al. Hereditary ataxia and spastic paraplegia in Portugal: a population- based prevalence study. JAMA Neurol. 2013 Jun;70(6):746-55. doi:10.1001/jamaneurol.2013.1707.

15. Wardle M, Morris HR, Robertson NP. Clinical and genetic characteristics of non-Asian dentatorubral-pallidoluysian atrophy: a systematic review. Mov Disord. 2009 Aug 15;24(11):1636-40.

16. Munhoz RP, Bergeron C, Lang AE. Sporadic case of dentatorubral pallidoluysian atrophy with no CAG repeat expansion and no intranuclear inclusions. Mov Disord. 2004 May;19(5):580-3.

17. Takano H, Cancel G, Ikeuchi T, Lorenzetti D, Mawad R, Stevanin $\mathrm{G}$, et al. Close associations between prevalences of dominantly inherited spinocerebellar ataxias with CAG-repeat expansions and frequencies of large normal CAG alleles in Japanese and Caucasian populations. Am J Hum Genet. 1998;63:1060-6.

18. Veneziano L, Frontali M. DRPLA. 1999 Aug 6 [Updated 2016 Jun 9]. In: Pagon RA, Adam MP, Ardinger HH, et al., editors. GeneReviews ${ }^{\circledR}$ [Internet]. Seattle (WA): University of Washington, Seattle; 1993-2016. Available from: https://www. ncbi.nlm.nih.gov/books/NBK1491/ 